\title{
Bit rate bound on superluminal communication
}

\author{
Xi Tong, ${ }^{1,2, *}$ Yi Wang $\odot,{ }^{1,2, \dagger}$ and Yuhang $\mathrm{Zhu}^{1,2, \$}$ \\ ${ }^{1}$ Department of Physics, The Hong Kong University of Science and Technology, \\ Clear Water Bay, Kowloon, Hong Kong, People's Republic of China \\ ${ }^{2}$ The HKUST Jockey Club Institute for Advanced Study, The Hong Kong University of Science \\ and Technology, Clear Water Bay, Kowloon, Hong Kong, People's Republic of China
}

(Received 13 January 2021; accepted 16 September 2021; published 15 October 2021)

\begin{abstract}
We study semiclassical communication in positivity-violating $k$-essence scalar field theories, with superluminal modes propagating on a rolling background. The self-interactions due to the nonlinear nature of these theories pose a constraint on the rate of superluminal information transfer. We derive a novel bit rate bound on superluminal communication within a conceptual model, to which a general class of $k$-essence theories reduces. Our result implies the possibility that, even if these positivity-violating $k$-essence theories may not possess a maximal information propagation speed, there is nevertheless an upper bound on the rate of information transfer.
\end{abstract}

DOI: 10.1103/PhysRevD.104.084062

\section{INTRODUCTION}

Special relativity $[1,2]$ is undoubtedly one of the most precise and concise theories of physics. Arising from its two underlying principles is a profound spacetime symmetry known as Lorentz invariance (LI). The causal structure of spacetime compatible with LI thus prohibits any superluminal motion that carries information [3-6], and the speed of light in the vacuum $c=1$ becomes the universal upper bound for information propagation. When consistently quantized, LI also puts tight constraints on the matter sector [7-12]. Experimentwise, decades of search for signs of Lorentz violation has only succeeded in improving its accuracy [13-17]. In the case with gravity, even though LI is not a strict isometry of the universe, it is still important in the sense of local inertial frames. At small distances, the spacetime curvature is subdominant, and physics that survives the decoupling limit $M_{p} \rightarrow \infty$ approaches that of the Minkowski spacetime. Moreover, the Lorentz-invariant causal structure of the local inertial frame is inherited in general relativity. Yet despite the great success of relativity, it remains a possibility, if not an elegant one by today's standards, that there exists a Lorentz-violating dark sector so weakly coupled to the

\footnotetext{
*xtongac@connect.ust.hk

phyw@ust.hk

yzhucc@connect.ust.hk
}

Published by the American Physical Society under the terms of the Creative Commons Attribution 4.0 International license. Further distribution of this work must maintain attribution to the author(s) and the published article's title, journal citation, and DOI. Funded by SCOAP ${ }^{3}$.
Standard Model (SM) that all the present experiments have not yet unveiled. Without the constraint of LI, the speed limit may be alleviated, possibly even indefinitely.

It has been known for a long time that certain families of scalar field theories admit superluminal modes when expanded upon an IR background [18-22], which spontaneously breaks LI (for similar superluminality setups in different contexts, see [23-26]). These $k$-essence scalars, although equipped with a formally local and Lorentzinvariant Lagrangian, are secretly nonlocal macroscopically [27]. The wrong signs in front of the higher-derivative operators in their Lagrangian forbid any UV completion satisfying the usual axioms of $S$-matrix theory. However, an open mind towards this is that, gravity aside [28], these unusual IR superluminal $k$-essence theories may not need to have a usual UV completion satisfying locality, analyticity, unitarity, and LI. They stand on their own as consistent IR theories respecting a weaker notion of causality [29-31], with a novel UV description unlike anything we have developed before. For instance, in nonstandard UV completions such as the qubit models $[32,33], \mathrm{LI}$ is not guaranteed in the UV and different fields can propagate at different speeds [32]. It is then not hard to imagine that on the lattice, a field belonging to the dark sector has a sound speed different from that of the SM fields, and is described at long distances by a positivityviolating $k$-essence Lagrangian. Therefore, it is nevertheless useful to understand their novelty within the IR region as a first step, namely, the exciting possibility of superluminal communication.

The reason for such a study is twofold. On one hand, superluminal modes, if exist, can be used to transmit information, which can be technologically useful. 
For instance, the $k$-essence field may live in the dark sector as a potential dark "Aether" for communication. The protocol for this superluminal-communication channel is thus necessary. On the other hand, if indeed a universal speed bound for information transfer is relaxed and locality/LI is forsaken, nature might still pose a universal bit rate bound for information transfer. As we will show in this paper, a bound on the bit rate of sending information can be derived from inspecting the behavior of superluminal $k$-essence theories in the IR.

\section{II. $\boldsymbol{k}$-ESSENCE AND SUPERLUMINALITY}

We begin with a lighting review of superluminality in $k$-essence theories [18-22]. Consider a scalar field (the $k$-essence) protected by a shift symmetry $\phi \rightarrow \phi+$ const. The Lagrangian $\mathcal{L}(X)$ is purely ${ }^{1}$ a function of $X=$ $-\frac{1}{2}(\partial \phi)^{2}$. The equation of motion (EoM) of $\phi$ is

$$
G^{\mu \nu} \nabla_{\mu} \nabla_{\nu} \phi=0
$$

where $\nabla_{\mu}$ is the covariant derivative compatible with the original spacetime metric $g_{\mu \nu}$, while small fluctuations around a fixed background [49] live effectively on an emergent geometry [50] defined by $G^{\mu \nu}=c_{s} \mathcal{L}_{{ }_{X}}^{-2}\left(\mathcal{L}_{, X} g^{\mu \nu}-\right.$ $\left.\mathcal{L}_{, X X} \nabla^{\mu} \phi \nabla^{\nu} \phi\right)$, with sound speed

$$
c_{s}^{-2}=1+2 X \frac{\mathcal{L}_{, X X}}{\mathcal{L}_{, X}} .
$$

This is most easily seen by expanding $\phi=\phi_{0}+\varphi$, where $\phi_{0}$ is a solution to the EoM of on-shell evolution. The perturbation evolves as

$$
G^{\mu \nu} D_{\mu} D_{\nu} \varphi=0
$$

Here $D$ is the covariant derivative calculated from the emergent metric $G$.

The sound speed $c_{s}$ can be either subluminal or superluminal, depending on the sign of $\mathcal{L}_{X X}$. The subluminal branch may enjoy a conventional UV completion. The superluminal branch, however, is generally believed to have no traditional UV completions in the absence of gravity [28], because it violates the positivity bound, which is a direct consequence of locality, analyticity, unitarity, and LI in the UV [27,35,51,52]. In the presence of gravity, the $t$-channel pole of graviton exchange diagrams poses a threat to the positivity bounds in the standard form [53]. This can result in a mild violation of the positivity bound

\footnotetext{
${ }^{1}$ Note that this is not the only healthy scalar theory with a shift symmetry. Higher-order derivative theories such as the Horndeski theory [34] (also known as generalized Galileons [35-38]) or Beyond Horndeski theories [39-44] are also ghost-free and shift symmetric. We choose $k$-essence both because it is technically simple and because it is well motivated in cosmology [45-48].
}

suppressed by $M_{p}^{-2}$, and therefore slight superluminality on curved spacetime backgrounds [54]. Although it is argued that this type of $t$-channel-pole superluminality is unresolvable in the validity range of effective field theory (EFT) and vanishes in the decoupling limit $M_{p} \rightarrow \infty$, we see that it is not impossible to violate positivity constraints in certain contexts (here, gravitation). Hence one still cannot completely rule out the possibility of some more severe violation of positivity and superluminality for other contexts. In fact, superluminality in the IR must stem from the highly nontrivial UV physics. For instance, in qubit models, both QED and gravity can emerge from the UV picture of quantum rotors placed on a lattice $[32,33]$. Such a nonstandard UV completion preserves locality, causality, and unitarity, but LI is not guaranteed: different fields can have different sound speeds [32]. Then it may be possible that a positivity-violating $k$-essence EFT admits such an UV embedding. Notice that although $\varphi$ travels outside the light cone, a restricted notion of causality is still respected under mild conditions of the background. Namely, there is a global time function associated with the rest frame of the background and therefore the IR EFT can be made free of paradoxical closed causal curves [31].

A complete theory of $k$-essence also includes nonlinear terms. One useful way to introduce nonlinearity is by defining the EFT as a theory only for perturbations in $X$. Namely, the EFT tower is built around a specific classical background:

$$
\mathcal{L}(X)=\sum_{n=0}^{\infty} \frac{1}{n !} \partial_{X}^{n} \mathcal{L}\left(X_{0}\right)\left(X-X_{0}\right)^{n}
$$

where $\partial_{X}^{n} \mathcal{L} \equiv \frac{c_{n}}{\Lambda^{4 n-4}}$. The validity of such an expansion is controlled by the energy of the perturbations only. In general, the renormalization procedure may bring new shift-symmetric operators that contain higher-order derivatives on the scalar field such as $\square^{n} \varphi$. In cases where the $k$-essence model can be entirely embedded to an UV theory like the nonlinear sigma model or the Dirac-Born-Infeld (DBI) Lagrangian [27,55], these terms will not appear. Although we are considering positivity-violating $k$-essence models without such UV completions, we still assume that these terms are absent and the EoM remains second order, so that the disastrous Ostrogradski ghosts are absent.

As a further simplification, we will study the rigid limit of Minkowski spacetime and decouple gravity by sending $M_{p} \rightarrow \infty$. Taking $\phi_{0}=\dot{\phi}_{0} t$ and $X_{0}=\frac{\dot{\phi}_{0}^{2}}{2}$, we then collect the leading interactions for $\varphi$ :

$$
\mathcal{L}(X)=(\text { total derivatives })+\mathcal{L}^{(2)}+\mathcal{L}_{\text {int }},
$$

with 


$$
\begin{gathered}
\mathcal{L}^{(2)} \equiv \frac{1}{2}\left[\left(c_{1}+\frac{c_{2} \dot{\phi}_{0}^{2}}{2 \Lambda^{4}}\right) \dot{\varphi}^{2}-c_{1}(\nabla \varphi)^{2}\right] \\
\mathcal{L}_{\text {int }} \equiv\left(\frac{c_{2} \dot{\phi}_{0}}{2 \Lambda^{4}}+\frac{c_{3} \dot{\phi}_{0}^{3}}{6 \Lambda^{8}}\right) \dot{\varphi}^{3}-\frac{c_{2} \dot{\phi}_{0}}{2 \Lambda^{4}} \dot{\varphi}(\nabla \varphi)^{2}+\cdots
\end{gathered}
$$

The sound speed is in agreement with (2). Being an EFT, the interacting Lagrangian (7) contains an infinite tower of higher-derivative terms. We will mainly focus on two leading interactions in the large- $c_{s}$ limit, namely $\frac{\eta}{\dot{\phi}_{0}} \dot{\varphi}^{3}$ and $\frac{\eta}{\dot{\phi}_{0}} \dot{\varphi}(\nabla \varphi)^{2}$, where $\eta \equiv \frac{c_{2} \dot{\phi}_{0}^{2}}{2 \Lambda^{4}}$. The reason why these two terms are relevant is as follows. Assuming the naturalness of the Wilson coefficients [i.e., $c_{n} \sim \mathcal{O}(1)$ ], the $c_{2}$ term should not be canceled by the $c_{3}$ term in the bracket before the interaction $\dot{\varphi}^{3}$ in (7). Its size sets the natural minimal amount of interactions. On the other hand, the $\dot{\varphi}(\nabla \varphi)^{2}$ term is always present whenever there is superluminality, and it is not contaminated by any (model-dependent) higher Wilson coefficients $c_{n}$ with $n \geq 3$. Thus its size sets the intrinsic minimal amount of interactions.

\section{BIT RATE BOUND}

Such superluminality of perturbations is in conflict with the notion that $c=1$ is the maximal speed of information transfer. Furthermore, the information propagation speed $c_{s}$ increases indefinitely with stronger background field strength while maintaining causality. Naively, there appears to be no upper limit to our ability to transfer information, because neither the speed nor capacity of the flow of information seems to receive any restriction.

This is the case if one only considers the quadratic part $\mathcal{L}^{(2)}$, since it gives rise to a linear wave equation without dispersion and solutions describing a trivial shapepreserving translation, $\varphi=\varphi\left(x \mp c_{s} t\right)$. However, one should be reminded that superluminality is a feature of the $k$-essence model (4) as a whole. In the complete picture, the seemingly unlimited ability of communication is weakened. For even if we can consistently let $c_{s} \rightarrow \infty$, we still run into the inevitable self-interaction of the perturbations whose intensity grows with the information content. This fact turns out to set an upper bound on the bit rate of superluminal communication. As we will demonstrate below, given the ability to perform superluminal communication with unlimited speed, one still has a limited bit rate of information transfer.

Let us consider the large- $c_{s}$ limit. Our goal is to find an constraint on the bit rate by considering nonlinearities of the EFT tower (7). In fact, the more interaction terms we include, the faster the signal gets distorted; hence, we acquire a smaller bit rate capacity. Therefore by considering one or two major interaction terms in the infinite EFT tower, we obtain an upper bound on the bit rate. The leading interactions, as argued above, are given by $\frac{\eta}{\dot{\phi}_{0}} \dot{\varphi}^{3}$ and $\frac{\eta}{\dot{\phi}_{0}} \dot{\varphi}(\nabla \varphi)^{2}$. We require them to be smaller than the quadratic Lagrangian $^{2}$ :

$\mathcal{L}_{\text {int }} \sim\left\{\left|\frac{\eta}{\dot{\phi}_{0}} \dot{\varphi}^{3}\right|,\left|\frac{\eta}{\dot{\phi}_{0}} \dot{\varphi}(\nabla \varphi)^{2}\right|\right\} \lesssim \mathcal{L}^{(2)} \sim \frac{1}{2}(1+\eta) \dot{\varphi}^{2}$,

where we have taken $c_{1} \approx 1$. This translates into a constraint on the perturbation field gradient,

$$
|\dot{\varphi}| \lesssim \frac{\left|\dot{\phi}_{0}\right|}{2 c_{s}^{p}}, \quad p=0,2
$$

where $p$ represents different interaction choices. $\frac{\eta}{\dot{\phi}_{0}} \dot{\varphi}^{3}$ giving rise to $p=2$ is based on assumption of naturalness. $p=0$ results from $\frac{\eta}{\dot{\phi}_{0}} \dot{\varphi}(\nabla \varphi)^{2}$ and is intrinsic. This inequality then translates into a constraint on the canonical energy (flux) density of free perturbations,

$$
\left|\left(T^{(2)}\right)^{0}{ }_{0}\right| \sim \frac{\dot{\varphi}^{2}}{c_{s}^{2}} \lesssim \frac{\dot{\phi}_{0}^{2}}{c_{s}^{2 p+2}}, \quad\left|\left(T^{(2)}\right)^{0}{ }_{i}\right| \sim \frac{\left|\dot{\varphi} \partial_{i} \varphi\right|}{c_{s}^{2}} \lesssim \frac{\dot{\phi}_{0}^{2}}{c_{s}^{2 p+3}},
$$

where we have neglected all $\mathcal{O}(1)$ constants. In the classical theory, a constraint on the energy flux is all there is to say about the system. However, upon quantization, the constraint on the energy flux automatically becomes a constraint on the information flux, since the energy of the signal is quantized in units of massless field quanta, which carries the encoded information.

For concreteness, let us set up a toy model for superluminal communication using $k$-essence perturbations. As a leading-order approximation, the nonlinearity will be turned down except when we evoke the constraint (9). Before discussing the details, we first point out that our communication model is essentially semiclassical in the sense that it does not entail the entanglement of quantum states. The information carried by the perturbations $\varphi$ resembles a classical analog signal carried, for example, by electromagnetic waves transmitted between a pair of parabolic dish antenna in point-to-point telecommunications.

Suppose there are two local observers (A and B) separated in the $x$ direction (see Fig. 1). The region between $\mathrm{A}$ and $\mathrm{B}$ is permeated by the background field $\phi_{0}$ which serves as a Aether-like medium for the superluminal $\varphi$ modes. A is equipped with an encoder capable of generating semiclassical signals according to the input information. $\mathrm{B}$ receives the signal and extract its information via a decoder. Since we are focusing on semiclassical

\footnotetext{
${ }^{2}$ Note that we are actually comparing the size of interactions on the level of the classical EoM. For this simple system, however, the constraint from EoM straightforwardly translates to the constraint of the Lagrangian (8) (i.e., after multiplying $\varphi$ and performing an integration-by-part).
} 


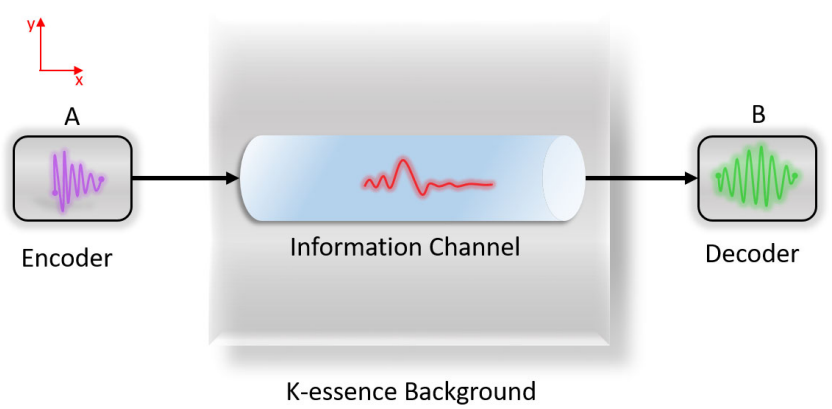

FIG. 1. An illustration of our superluminal communication model.

superluminal communication, the natural choice for the signal is a coherent state [56,57],

$$
|z\rangle=\exp \left(-\frac{1}{2} \int_{\vec{k}}\left|z_{\vec{k}}\right|^{2}\right) \exp \left(\int_{\vec{k}} z_{\vec{k}} a_{\vec{k}}^{\dagger}\right)|0\rangle
$$

where $z_{\vec{k}}$ is the eigenvalue of the annihilation operator, $a_{\vec{k}}|z\rangle=z_{\vec{k}}|z\rangle$. The information content of the signal is encoded in the Fourier coefficients $\left\{z_{\vec{k}}\right\}$. Generically for point-to-point communications, the signal takes the form of a wave packet with IR cutoff scales $L_{x}, L_{y}$, and $L_{z}$. In addition, to maximize the efficiency of communication, the wave packet should be traveling roughly in the $+x$ direction. Hence the Fourier components along the transverse directions should be turned down, with an uncertainty of $\Delta k_{y} \sim \frac{1}{L_{y}}, \Delta k_{z} \sim \frac{1}{L_{z}}$. In other words, we impose

$$
z_{\vec{k}}=0 \quad \text { for }\left|k_{y}\right|>\frac{2 \pi}{L_{y}} \quad \text { or } \quad\left|k_{z}\right|>\frac{2 \pi}{L_{z}} .
$$

By this choice, $z_{\vec{k}}$ only has support for $\vec{k}$ lying on the $+x$ direction; then the evolution of the signal is simplified to a shape-preserving translation, i.e., $\varphi=\varphi\left(x-c_{s} t\right)$. In this way we have reduced the communication model to a onedimensional problem while maintaining its maximal ability to transfer information. We define the expectation value of the area density of energy by

$$
E[\hat{z}] \equiv \frac{\left\langle z\left|H^{(2)}\right| z\right\rangle}{L_{y} L_{z}}=\int \frac{d k}{2 \pi} \omega_{k}\left|\hat{z}_{k}\right|^{2}
$$

where $\hat{z}_{k} \equiv \frac{z_{k}}{L_{y} L_{z}}$ is the reduced Fourier moment. In order to emit the signal, we turn on a weak coupling between the $k$-essence perturbation $\varphi$ and the visible SM sector via a shift-symmetric coupling $J_{\mathrm{SM}}^{\mu} \partial_{\mu} \varphi$. The source $J_{\mathrm{SM}}^{\mu}$ is turned on for $0<t<\frac{L_{x}}{c_{s}}$. In the free-theory limit, this can be mimicked by imposing a periodic boundary condition between the sound cones originating from the source, leading to momentum quantization $k_{n}=\frac{2 \pi n}{L_{x}}$. Notice that this is only possible in the free-theory limit. In the presence of interactions, there is no automatic boundary condition inherited from the source function and the wave packet will in general spread out due to its self-interactions.

With all the preparations complete, now we can apply the constraint (9) on the field gradient to obtain our bit rate bound. In the semiclassical limit, the information content of the communication channel where the messages are sent according to a given probability distribution functional $P[\hat{z}]$ can be characterized by the Shannon entropy:

$S[P]=-\int \mathcal{D}^{2} \hat{z} P[\hat{z}] \ln P[\hat{z}]$, with $\int \mathcal{D}^{2} \hat{z} P[\hat{z}]=1$.

The integration measure $\mathcal{D}^{2} \hat{z} \propto \prod_{k>0} d \hat{z}_{k} d \hat{z}_{k}^{*}$ must be taken with a grain of salt. The fact that the coherent state basis is overcomplete [56] leads to unphysical contributions to the Shannon entropy. These contributions must be gauged away by truncating the phase-space integral near the origin. Equivalently, one can start with the von Neumann entropy $S_{\mathrm{V} . \mathrm{N} .}[P]=-\operatorname{Tr} \rho \ln \rho$ of a density matrix $\rho[P]=\int \mathcal{D}^{2} \hat{z} P[\hat{z}]|z\rangle\langle z|$ describing a statistical mixture of coded signals.

We can impose three conceivable constraints. The most straightforward one from (9) is a local constraint imposed at every spacetime point throughout the interior of the wave packet,

$(\mathrm{LC}):|\langle z|\dot{\varphi}(t, x)| z\rangle| \lesssim \frac{\left|\dot{\phi}_{0}\right|}{c_{s}^{2 p}} \quad$ for $c_{s} t-L_{x}<x<c_{s} t$.

This is too restrictive since (LC) violations may not endanger the global information content of the signal. The second is a global constraint that smears (LC) over the wave packet using (10) and (13):

$$
(\mathrm{GC}): E[\hat{z}] \lesssim \varepsilon_{\max } L_{x}
$$

where $\varepsilon_{\max } \equiv\left|\left(T^{(2)}\right)^{0}{ }_{0}\right|_{\max } \sim \frac{\dot{\phi}_{0}^{2}}{c_{s}^{2 p+2}}$ is the maximal energy volume density. The third is an average global constraint:

$$
(\mathrm{AGC}): \int \mathcal{D}^{2} \hat{z} P[\hat{z}] E[\hat{z}] \lesssim \varepsilon_{\max } L_{x} .
$$

Namely, we do not require (GC) to be satisfied by all signals generated from $P[\hat{z}]$, but only by its average output. This is also more suitable compared to (GC), in the sense that the failure of selective signals does not affect the efficiency of the whole coding method $P[\hat{z}]$. The violation of (AGC) would imply nonlinearity so large that signals cannot be prepared or even defined within the theory (7), i.e., an IR breakdown of the EFT. It is useful to notice the inclusion relation between the constraints, 


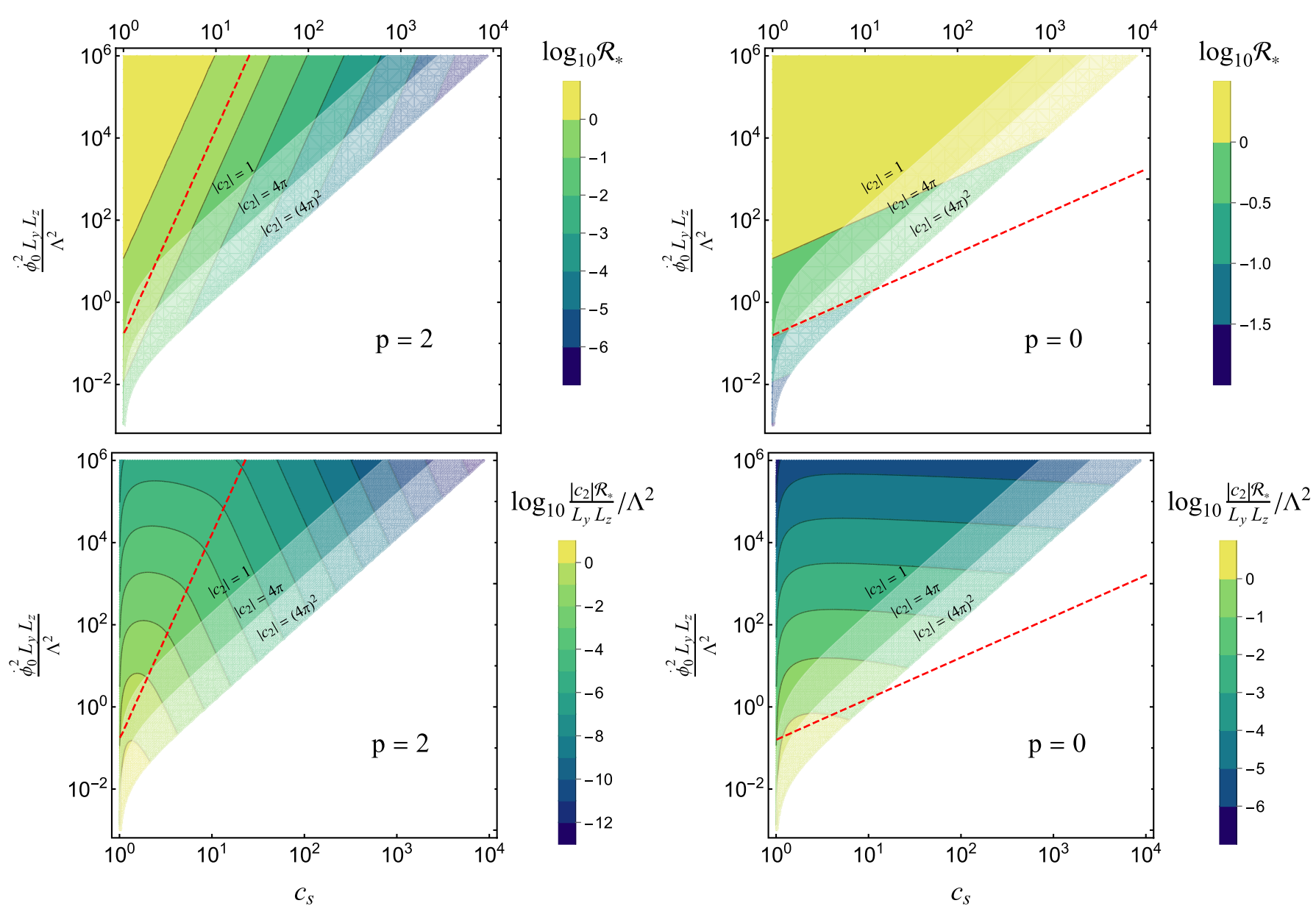

FIG. 2. Dimensionless bit rate bound $\mathcal{R}_{*}$ (first row) and its area density (second row). The left column is the natural bound corresponding to $p=2$ while the right column is the intrinsic bound with $p=0$, which is looser but more general. The red dashed line is given by $\frac{\dot{\phi}_{0}^{2} L_{y} L_{z}}{\Lambda^{2}}=\frac{c_{s}^{2 p+1}}{2 \pi}$. The region above this line is the logarithmic high-temperature case while that below the line is the power-law lowtemperature case. The lower regions are excluded due to EFT validity for given choices of $\left|c_{2}\right|$.

Tightness: $(\mathrm{LC})>(\mathrm{GC}) \geq(\mathrm{AGC})$.

Before we continue on applying (AGC), we stress that the reason why we choose to constrain $H=\left(T^{(2)}\right)^{0}{ }_{0}$ is not because we wish to couple $k$-essence to gravity, but because it is easy to calculate when compared to the (LC) constraint on $|\dot{\varphi}|$. In terms of signal encoding and preparation, it is also more relevant.

Now it is straightforward to bound the Shannon entropy by maximizing it under (AGC). Introducing two Lagrange multipliers $\alpha, \beta$, we seek the stationary point by

$$
0=\delta\left(S[P]-(\alpha-1) \int \mathcal{D}^{2} \hat{z} P-\beta \int \mathcal{D}^{2} \hat{z} P E\right)
$$

The optimal probability distribution then takes a familiar form, $P_{*}=e^{-\alpha-\beta E}$. The Lagrange multipliers, like in thermodynamics, are solved from the constraints. The maximal entropy is then

$$
S_{*}=\frac{L_{x} \Lambda}{2 \pi c_{s}} \ln \left(1+\frac{1}{\gamma} e^{-\gamma}\right)+\frac{2 \dot{\phi}_{0}^{2} L_{x} L_{y} L_{z}}{c_{s}^{2 p+2} \Lambda} \gamma,
$$

where $\gamma$ is the solution to

$$
\frac{1}{\gamma^{2}} \int_{0}^{\gamma} d x \frac{1+x}{1+x e^{x}}=\frac{2 \pi \dot{\phi}_{0}^{2} L_{y} L_{z}}{c_{s}^{2 p+1} \Lambda^{2}}
$$

Thus the (AGC) bound for bit rate is given by

$$
R_{*}=\frac{c_{s} S_{*}}{L_{x}} \equiv \Lambda \mathcal{R}_{*}\left(c_{s}, \dot{\phi}_{0}^{2} L_{y} L_{z} / \Lambda^{2}\right) .
$$

This bit rate bound simplifies considerably in two limits. In the small cross-section limit where $\frac{\dot{\phi}_{0}^{2} L_{y} L_{z}}{\Lambda^{2}} \ll \frac{c_{s}^{2 p+1}}{2 \pi}$, the bit rate bound is independent of $\Lambda$, 


$$
R_{*} \approx\left(\frac{2.64}{\pi} \dot{\phi}_{0}^{2} L_{y} L_{z}\right)^{1 / 2} c_{s}^{-(2 p+1) / 2}
$$

On the other hand, if the communication cross section is large, i.e., $\frac{\dot{\phi}_{0}^{2} L_{y} L_{z}}{\Lambda^{2}} \gg \frac{c_{s}^{2 p+1}}{2 \pi}$, the EFT cutoff $\Lambda$ explicitly appears in the bit rate bound,

$$
R_{*} \approx \frac{\Lambda}{2 \pi} \ln \frac{2 \pi \dot{\phi}_{0}^{2} L_{y} L_{z}}{c_{s}^{2 p+1} \Lambda^{2}} .
$$

The full dependence of the dimensionless bit rate $\mathcal{R}_{*}$ as well as its area density is shown in Fig. 2. Obviously it is monotonically decreasing with $c_{s}$. As a result, increasing the propagation speed indefinitely cannot always lead to an increasing bit rate, since its upper bound drops with $c_{s}$. Moreover, although mathematically $\mathcal{R}_{*}$ is not bounded from above due to its logarithmic growth with the crosssection area $L_{y} L_{z}$, the bit rate area density is, however, decreasing with $L_{y} L_{z}$. Therefore, the bit rate area density is universally bounded by

$\frac{R_{*}}{L_{y} L_{z}} \lesssim\left(\frac{2.64}{\pi}\right)^{1 / 2} \dot{\phi}_{0} \Lambda c_{s}^{-\frac{2 p+3}{2}} \approx 1.30\left|c_{2}\right|^{-1 / 2} c_{s}^{-\frac{2 p+3}{2}} \Lambda^{3}$,

where we have set a minimal area $L_{y} L_{z} \sim c_{s}^{2} \Lambda^{-2}$ consistent with the EFT in (23). Then (25) gives a maximal ability to compress the signal in the transverse direction. ${ }^{3}$ Namely, bit rate per unit area is still bounded.

We point out that our treatment resembles the description of a one-dimensional photon gas confined in a box with a modified dispersion relation. The small cross-section case corresponds to its low-temperature behavior, where highfrequency modes are automatically suppressed by the Boltzmann factor and the cutoff frequency $\Lambda$ is never reached. The large cross-section limit corresponds to the high-temperature case, where modes near the cutoff can also be excited with abundance. But this time, the validity of EFT puts a sharp cutoff to the available modes, and the final result is dependent on $\Lambda$, reminiscent of the UV catastrophe in classical physics.

\footnotetext{
${ }^{3}$ In free space, too much restriction in the transverse direction generically leads to a fast-spreading wave packet. This can be prevented, for example, by using a narrow waveguide, where transverse excitations are energetically too expensive to be excited.
}

There are, of course, two important distinctions. The first one being conceptual, is that we are not actually sending a thermal mixture of states confined in a one-dimensional box. We have precise control over the signal, which is in a pure state. The entropy resides in the coding method we use. The maximal entropy under (AGC) is reached by coding the messages following a Boltzmann distribution. Of course, one does not have to code the messages this way. Freedom is given to the sender A such that any probabilistic distribution $P[\hat{z}]$ is allowed, although none of them can exceed the efficiency provided by $P_{*}[\hat{z}]$.

The second one being technical, is that we are counting coherent states of the $k$-essence field rather than their energy eigenstates (when confined to a box). The coherent states are almost classical, whereas the energy eigenstates are nonclassical. When the nonlinear interaction of $k$-essence is turned down, either choice is fine. In fact, a simple calculation shows that they give the same Shannon entropy $S_{*}$ up to an $\mathcal{O}(1)$ numerical factor.

\section{CONCLUSION}

In this paper we have investigated superluminal communication in a family of positivity-violating $k$-essence theories. After setting up a conceptual superluminal communication protocol, by the requirement of subdominant nonlinearity, we have derived a bit rate bound and a universal bit rate area density bound for all superluminal $k$-essence models. Notice that in this work we focus exclusively on the bit rate bound intrinsic to the $k$-essence theory itself. If such superluminal dark $k$-essence does exist, its leading-order influence on the visible sector would manifest itself as Lorentz violations in the SM. The phenomenological implications and a detailed analysis of the signal evolution are left for future works. Finally, we remark that $k$-essence is not the only superluminal communication candidate. It is interesting to consider whether similar bit rate bounds exist for other methods [58,59].

\section{ACKNOWLEDGMENTS}

We thank Ali Akil, Lingfeng Li, Kun-Feng Lyu, and Henry Tye for helpful discussions and suggestions. This work was supported in part by the NSFC Excellent Young Scientist (EYS) Scheme (Hong Kong and Macau) Grant No. 12022516. 
[1] A. Einstein, Ann.Phys. (Leipzig) 17, 891 (1905).

[2] A. Einstein, Ann.Phys. (Leipzig) 23, 371 (1907).

[3] L. Brillouin, Wave Propagation and Group Velocity (Academic Press, New York, 1960), Vol. 8.

[4] R. Fox, C. Kuper, and S. Lipson, Nature (London) 223, 597 (1969).

[5] M. J. Rees, Nature (London) 211, 468 (1966).

[6] T. M. Davis and C. H. Lineweaver, Pub. Astron. Soc. Aust. 21, 97 (2004).

[7] M. Fierz, Helv. Phys. Acta 12, 3 (1939).

[8] W. Pauli, Phys. Rev. 58, 716 (1940).

[9] G. Luders, Kong. Dan. Vid. Sel. Mat. Fys. Med. 28N5, 1 (1954).

[10] S. Weinberg, Phys. Rev. 135, B1049 (1964).

[11] S. R. Coleman and J. Mandula, Phys. Rev. 159, 1251 (1967).

[12] S. Weinberg and E. Witten, Phys. Lett. 96B, 59 (1980).

[13] D. Colladay and V. Kostelecky, Phys. Rev. D 58, 116002 (1998).

[14] S. R. Coleman and S. L. Glashow, Phys. Rev. D 59, 116008 (1999).

[15] V. Kostelecky and N. Russell, Rev. Mod. Phys. 83, 11 (2011).

[16] A. G. Cohen and S. L. Glashow, Phys. Rev. Lett. 107, 181803 (2011).

[17] S. Liberati, Classical Quantum Gravity 30, 133001 (2013).

[18] C. Armendariz-Picon, T. Damour, and V. F. Mukhanov, Phys. Lett. B 458, 209 (1999).

[19] J. Garriga and V.F. Mukhanov, Phys. Lett. B 458, 219 (1999).

[20] C. Armendariz-Picon, V. F. Mukhanov, and P. J. Steinhardt, Phys. Rev. Lett. 85, 4438 (2000).

[21] C. Armendariz-Picon, V. F. Mukhanov, and P. J. Steinhardt, Phys. Rev. D 63, 103510 (2001).

[22] V. F. Mukhanov and A. Vikman, J. Cosmol. Astropart. Phys. 02 (2006) 004.

[23] I. Drummond and S. Hathrell, Phys. Rev. D 22, 343 (1980).

[24] K. Scharnhorst, Phys. Lett. B 236, 354 (1990); 787, 204(E) (2018).

[25] G. Barton, Phys. Lett. B 237, 559 (1990).

[26] T. Jacobson and D. Mattingly, Phys. Rev. D 70, 024003 (2004).

[27] A. Adams, N. Arkani-Hamed, S. Dubovsky, A. Nicolis, and R. Rattazzi, J. High Energy Phys. 10 (2006) 014.

[28] G. Shore, Nucl. Phys. B778, 219 (2007).

[29] C. Armendariz-Picon and E. A. Lim, J. Cosmol. Astropart. Phys. 08 (2005) 007.

[30] J.-P. Bruneton, Phys. Rev. D 75, 085013 (2007).

[31] E. Babichev, V. Mukhanov, and A. Vikman, J. High Energy Phys. 02 (2008) 101.
[32] M. Levin and X.-G. Wen, Phys. Rev. B 73, 035122 (2006).

[33] Z.-C. Gu and X.-G. Wen, Nucl. Phys. B863, 90 (2012).

[34] G. W. Horndeski, Int. J. Theor. Phys. 10, 363 (1974).

[35] A. Nicolis, R. Rattazzi, and E. Trincherini, J. High Energy Phys. 05 (2010) 095; 11 (2011) 128(E).

[36] C. Deffayet, G. Esposito-Farese, and A. Vikman, Phys. Rev. D 79, 084003 (2009).

[37] C. Deffayet, X. Gao, D. A. Steer, and G. Zahariade, Phys. Rev. D 84, 064039 (2011).

[38] T. Kobayashi, M. Yamaguchi, and J. Yokoyama, Prog. Theor. Phys. 126, 511 (2011).

[39] J. Gleyzes, D. Langlois, F. Piazza, and F. Vernizzi, Phys. Rev. Lett. 114, 211101 (2015).

[40] J. Gleyzes, D. Langlois, F. Piazza, and F. Vernizzi, J. Cosmol. Astropart. Phys. 02 (2015) 018.

[41] D. Langlois and K. Noui, J. Cosmol. Astropart. Phys. 02 (2016) 034.

[42] J. Ben Achour, M. Crisostomi, K. Koyama, D. Langlois, K. Noui, and G. Tasinato, J. High Energy Phys. 12 (2016) 100.

[43] M. Crisostomi, K. Koyama, and G. Tasinato, J. Cosmol. Astropart. Phys. 04 (2016) 044.

[44] J. B. Achour, D. Langlois, and K. Noui, Phys. Rev. D 93, 124005 (2016).

[45] C. Armendariz-Picon, T. Damour, and V. F. Mukhanov, Phys. Lett. B 458, 209 (1999).

[46] T. Chiba, T. Okabe, and M. Yamaguchi, Phys. Rev. D 62, 023511 (2000).

[47] C. Armendariz-Picon, V. F. Mukhanov, and P. J. Steinhardt, Phys. Rev. Lett. 85, 4438 (2000).

[48] C. Armendariz-Picon, V. F. Mukhanov, and P. J. Steinhardt, Phys. Rev. D 63, 103510 (2001).

[49] R. M. Wald, General Relativity ( Chicago University Press, Chicago, 1984).

[50] C. Barcelo, S. Liberati, and M. Visser, Living Rev. Relativity 8, 12 (2005).

[51] T. Pham and T. N. Truong, Phys. Rev. D 31, 3027 (1985).

[52] C. de Rham, S. Melville, A. J. Tolley, and S.-Y. Zhou, Phys. Rev. D 96, 081702 (2017).

[53] L. Alberte, C. de Rham, S. Jaitly, and A. J. Tolley, Phys. Rev. D 102, 125023 (2020).

[54] C. de Rham and A. J. Tolley, Phys. Rev. D 102, 084048 (2020).

[55] S. Mukohyama and R. Namba, J. Cosmol. Astropart. Phys. 02 (2021) 001.

[56] J. R. Klauder, Ann. Phys. (N.Y.) 11, 123 (1960).

[57] R. J. Glauber, Phys. Rev. 131, 2766 (1963).

[58] S. S. Gubser, Phys. Lett. B 705, 279 (2011).

[59] R. Ramos, D. Spierings, I. Racicot, and A. M. Steinberg, Nature (London) 583, 529 (2020). 